\title{
Bem-estar durante o período gestacional de ovelhas: uma breve revisão
}

\author{
Welfare during gestational period of ewes: a short review
}

Henrique Barbosa Hooper, Fábio Luís Henrique, Lina Fernanda Pulido Rodríguez, Cristiane Gonçalves Titto

Universidade de São Paulo (USP), Pirassununga, SP, Brasil

\section{Resumo}

Nesta revisão, objetiva-se abordar práticas e cuidados relacionados ao bem-estar de ovelhas durante a gestação em suas fases distintas. 0 bem-estar tem sido foco de estudos em diversas áreas na ovinocultura, mas pouco se tem discutido a respeito de boas práticas durante o período gestacional. A gestação é um ponto-chave para produtividade e rentabilidade da ovinocultura, e os bons cuidados neste período podem evitar problemas futuros. Dessa forma, são abordados aspectos principais do comportamento ovino, pontos relacionados à reprodução, gestação e relação materno-filial. Também são discutidos os impactos do estresse pré-natal nas funções fisiológicas, neuroendócrinas, reprodutivas e de desempenho dos cordeiros. Estas considerações permitirão o melhor entendimento do bem-estar positivo das ovelhas gestantes por meio de aspectos nutricionais, ambientais e de manejo que favorecem a gestação, o parto e o nascimento de cordeiros saudáveis.

Palavras-chave: Cordeiro. Estresse Pré-Natal. Ovino. Comportamento Materno-Filial.

\begin{abstract}
In this review, practices and cares related to welfare of ewes during gestation in its distinct phases are reported. Welfare has been the focus of studies in several areas on sheep production, nevertheless little has been discussed about the good practices during the gestational period. Gestation is a key point for productivity and profitability of sheep systems and the good cares in this period can avoid future problems. Hence, the main aspects of ewe behavior, reproduction, gestation and maternal-filial relationship are discussed. As well as the impacts of prenatal stress on the physiological, neuroendocrine, reproductive and performance functions of lambs. These considerations will provide a better understanding of the positive welfare of the pregnant ewes in view of nutritional, environmental and management aspects that contribute to gestation, delivery and the birth of healthy lambs.
\end{abstract}

Keywords: Lamb. Prenatal Stress. Sheep. Maternal-Fetal Behavior. 


\section{Introdução}

Bem-estar animal é um tema cada vez mais discutido e estudado na área dos ruminantes. A intensificação dos sistemas de produção, a fim de aumentar a oferta de alimentos, evidenciou práticas de manejo que prejudicavam as necessidades e o comportamento natural dos animais (Fraser, 2008). Além do mais, o fenômeno da globalização acelerou a maneira de as pessoas obterem informação, mudando a percepção do que é consumido e a exigência por produtos de origem animal oriundos de criações éticas (Pinheiro e Brito, 2009; Freitas et al.; 2017). Desta forma, a relação entre humanos e animais está em constante foco de conduta moral e, por defrontar com preocupações éticas, é relevante entender e discutir boas práticas de manejo e cuidados nos diferentes ciclos produtivos.

$\mathrm{Na}$ ovinocultura, o bem-estar tem sido foco de estudos em diversas áreas, tais como a castração (Thornton e Waterman-Pearson, 2002), corte de cauda (Wang et al., 2018), tosquia (Rutherford, 2002), manipulação (Fitzpatrick et al., 2006), desmame (Henrique, 2017), transporte (Broom, 2005) e abate (More et al., 2017). No Brasil, a ovinocultura desempenha papel importante pela fixação do homem no campo e pela produção de alimentos e renda em regiões com pouca disponibilidade de terras agriculturáveis (Madruga et al., 2005). 0 rebanho nacional apresentou em 2016 um efetivo de 18,4 milhões de cabeças, com uma taxa de crescimento anual de 1,5\% entre os anos de 2011 e 2016 (IBGE, 2016).

Para o agronegócio nacional, a ovinocultura é uma opção de investimento devido à expansão do mercado nacional, condições climáticas para criação, disponibilidade de área, forragem e mãode-obra (Madruga et al., 2005). O cordeiro é a categoria animal com características cárneas com maior aceitabilidade pelos consumidores, menor ciclo produtivo, melhores características de carcaça e maior eficiência no ganho de peso (Pires et al., 2000). Para que um produto final com qualidade global chegue ao consumidor, diversas etapas produtivas são necessárias, desde a reprodução das ovelhas, gestação, aleitamento e desmame, até terminação, transporte e abate.

A gestação é um ponto chave dentro dessas etapas, e os bons cuidados e manejo da ovelha gestante são fundamentais na obtenção de cordeiros saudáveis. Sabe-se que durante a fase fetal e neonatal, o eixo hipotálamo-hipófise-adrenal está mais suscetível, e o estresse nestas fases pode acarretar alterações no desenvolvimento placentário, fetal, neural e neuroendócrino (Levine, 2001; Jensen et al., 2002; Levine, 2002; Matthews, 2002; Braun, 2017). Dessa forma, esta revisão abordará práticas e cuidados relacionados ao bem-estar de ovelhas durante a gestação em suas distintas fases.

\section{Entendendo o comportamento ovino}

A relação entre humanos e ovinos, uma das primeiras espécies domesticadas pelo homem, tem provavelmente mais de 6.000 anos (Hulet etal., 1975). Foi a partir de um ovino silvestre (Ovis orientalis), que vivia em montanhas nas regiões oeste e central da Ásia, que se iniciou a domesticação (CluttonBrock, 1999). 0 fato de os ovinos serem tolerantes ao frio, produzirem lã para vestimentas e carne para consumo contribuiu para sua disseminação. A relação homem-animal evoluiu da caça para a domesticação, consolidando os primeiros rebanhos e fazendas para carne e lã (Zohary et al., 1998).

Os ovinos são animais de comportamento visual, inteligentes evigilantes (Berger,1978). Considerados presas na natureza, detêm boa visão, com um ângulo aproximado de $270^{\circ}$ (Whitteridge, 1978). Vivem em grupo, o que lhes confere melhor proteção contra predadores, maior sobrevivência das crias e favorece o acasalamento (Berger, 1978; Festa-Bianchet, 1991; Menzies, 1994). Os grupos realizam as atividade diárias em conjunto. Como uma imitação sincronizada, eles caminham, pastejam, deitam, desviam de obstáculos, correm e bebem água juntos (Banks, 1964; Rech, 2008). O comportamento de seguir se desenvolve desde o nascimento, quando a cria segue a mãe nas atividades diárias (Rech, 2008). É importante salientar que dentro do grupo há a formação de subgrupos, mesmo que todos os animais permaneçam juntos no rebanho. Estes subgrupos realizam suas atividades em conjunto e os cordeiros aprendem a permanecer no mesmo subgrupo das mães, mantendo esta característica entre gerações. 
0 comportamento de intimidação ajuda a reduzir brigas e, por consequência, menor risco de injúrias. Quando este comportamento falha, porém, para estabeler a dominância e deter um rival, os ovinos se confrontam de cabeça baixa e pescoço esticado, batendo cabeça com cabeça (Keeling e Gonyou, 2001). 0 olfato é um importante meio de reconhecimento entre indivíduos, fato que explica o comportamento de cheirar novos membros dentro do grupo (Baldwin e Meese, 1977). Os ovinos detêm excelente habilidade de aprendizado, condicionamento, discriminação sensorial, aversão, resolvem bem testes de labirinto e apresentam memória de curto e longo prazo (Kendrick et al., 1996; Doyle et al., 2014).

Como ruminantes, eles pastejam em grupos, com forrageamento baixo, mantendo sempre uma pequena distância entre indivíduos. A distância durante o pastejo pode variar conforme a raça, estação do ano, topografia, qualidade e altura da vegetação, e distância da fonte de água (Banks, 1964; Lynch et al., 1992; Dumont e Boissy, 2000; Hopewell et al., 2005). Os mais independentes dentro do grupo e que pastejam mais distantes são os responsáveis por direcionar e movimentar o rebanho. 0 líder conduz as atividades, sendo seguido pelos outros componentes do grupo. Esta liderança depende do tamanho, composição e organização do grupo (Grandin, 2007).

A liderança é um comportamento adaptativo que não só auxilia o movimento do rebanho, como também o acesso a alimentos, o acasalamento e a solução de problemas sociais (Clutton-Brock et al., 1986; Pérez-Barbería et al., 2015). Pérez-Barbería e Walker (2018), ao monitorarem ovelhas Scottish Blackface com auxílio de GPS para melhor entender as dinâmicas das interações sociais entre os indivíduos do rebanho, descobriram que a posição de liderança relativa ao movimento do rebanho não é afetada pela condição reprodutiva das ovelhas e que a liderança individual de uma ovelha também não é afetada pelo parto e nascimento da cria.

\section{Aspectos reprodutivos e materno-filiais}

As ovelhas são consideradas animais poliéstricos estacionais, com acasalamento nas estações de dias curtos, outono e inverno (Rosa e Bryant, 2003). A fêmea tem média de duração do ciclo estral de 16,7 dias, cio de 18 a 24 horas, com aumento da atividade motora, inquietação e balidos (Banks, 1964; Hafez e Hafez, 2000). Os machos tendem a ficar mais agressivos e inquietos nesta época. Eles manifestam o comportamento de Flehmen, no qual, após distinguir a fêmea no cio, cheiram a urina, erguem a cabeça e curvam os lábios, permanecendo nesta posição por volta de 10 a 30 segundos (Banks, 1964). A corte termina quando a fêmea aceita imóvel o macho, assumindo posição que favorece a monta e acontecendo, então, a cópula.

No momento do parto, a fêmea gestante se afasta do rebanho (Rech, 2008), desejando um ambiente limpo, calmo e sem ruídos, onde possa parir tranquilamente (Arnold e Morgan, 1975). Dois meses antes do parto há o início das contrações uterinas de baixa amplitude e longa duração; com a proximidade do parto, há o aumento da amplitude e redução da duração das contrações (Fitzpatrick e Dobson 1979). Segundo os mesmo autores, na primeira fase do parto a frequência das contrações se torna regular, de curta duração e elevada amplitude. Quando a pressão intra-uterina se eleva para 30-40 $\mathrm{mmHg}$, a ovelha entra no segundo estágio do parto, com substancial elevação da frequência entre contrações, com pequenas pausas entre estas (Fitzpatrick e Dobson, 1979). De uma a duas horas antes da segunda fase do parto, ocorre a dilatação progressiva do canal cervical, vagina e vulva. Após a expulsão do feto, o cordão umbilical se rompe por estiramento, a mãe lambe a cria e o estimula a levantar; em algumas ocasiões ocorre a placentofagia (O'Connor e Lawrence, 1992; PérezTorrero e Rubio-Navarro, 2015).

Um estímulo importante logo após o nascimento é lamber o recém-nascido, o que permite a ovelha distinguir o seu cordeiro dos demais (Levy e Poindron, 1987). Este contato logo após o nascimento é de fundamental importância para a sobrevivência do cordeiro e para o aprimoramento da habilidade materna da mãe (Levine et al., 1958; Nowak, 2000). É neste ato que a mãe remove os fluídos placentários presentes no filhote, o que contribui para a retenção de calor, estimula-o a levantar e procurar o teto, além de aumentar o vínculo entre mãe e cria (Nowak e Boivin, 2015). 
Cordeiros expostos a situações adversas durante a gestação ou mesmo após o nascimento podem receber mais atenção por parte da ovelha, com o intuito de compensar de alguma maneira este revés (Hild et al., 2010).

A forte ligação entre mãe e filhote é estabelecida principalmente pelo olfato (Baldwin e Meese, 1977). A pesquisa de Alexander e Walser (1978) também evidenciou o reconhecimento visual como meio importante da relação materno-filial, principalmente pelo padrão de cores da cabeça do cordeiro. $\mathrm{O}$ balido do filhote pode ser reconhecido pela mãe, e a vocalização é a forma que a ovelha chama o cordeiro para mamar.

Ewbank (1967) percebeu que cordeiros gêmeos mamam mais frequentemente até o final da $4^{\circ}$ semana de vida em comparação com cordeiros únicos. 0 autor também afirmou que a duração das mamadas é semelhante entre cordeiros gêmeos, e nas três primeiras semanas há uma correlação entre cuidados maternais e mamada. Arnold et al. (1979) constataram que apesar de a produção de leite declinar com o avanço da lactação, a ligação entre mãe e cria permanece por mais de 100 dias. A duração e frequência de mamada declina à medida que a cria ganha peso, até o momento em que a ovelha passa a apresentar comportamento antagonístico para mamada, na qual ela dificulta a mamada do cordeiro com o movimento dos membros pélvicos sobre a glândula mamária e/ou deslocamento (Maldonado et al., 2015).

\section{Bem-estar durante a gestação}

Nossistemas de criação extensivo e semi-intensivo, os ovinos melhor manifestam comportamentos e hábitos naturais da espécie (Goddard et al., 2006; Stefanakis et al., 2007; Munoz et al., 2018). Isso não significa, contudo, que o grau de bem-estar não possa ser comprometido por algumas situações adversas, dentre as quais o desbalanço nutricional (Pereira et al., 2018), manejo, transporte e instalações inadequados (Fazio et al., 2018), privação de espaço (Caroprese, 2008), irregularidades no piso (Færevik et al., 2005), adversidades climáticas (Marai et al., 2007), comprometimento imunológico (Sevi e Caroprese, 2012) e parasitas (Kumar et al., 2017).
Nos cinco meses de gestação das ovelhas, distinguem-se três períodos principais (início, meio e final da gestação), e cada um exige cuidados especiais. Dessa forma, após confirmação da gestação, torna-se importante saber os períodos que as ovelhas irão requerer mais atenção.

0 começo da gestação (da concepção até os 40 dias) é um período crítico para a perda e reabsorção embrionária. Deve-se, portanto, evitar o desbalanço nutricional, manipulações excessivas, contenções, casqueamento, mistura de lotes e mudanças de ambiente, bem como qualquer medida que ocasione estresse. Nas primeiras duas semanas após a concepção, o embrião ainda está migrando no útero, onde recebe oxigênio e nutrientes. Na terceira semana, a placenta se desenvolve, e é neste momento que o embrião é designado feto (Hafez e Hafez, 2000).

Gestações múltiplas fazem com que os fetos compartilhem os nutrientes disponíveis, o que explica o nascimento de cordeiros menores (Gardner et al., 2007). Alguns estudos demonstram que a ovulação e as taxas de concepção e implantação do embrião melhoram com a realização da prática do flushing (Molle et al.,1995; Mori et al., 2006; Tur et al., 2017), estratégia nutricional que consiste em disponibilizar às fêmeas gestantes antes e após a estação reprodutiva um volumoso ou concentrado de qualidade (Naqvi et al., 2012). Evita-se, assim, que a ovelha perca escore de condição corporal, o que comprometeria a implantação do embrião.

0 meio da gestação $\left(40^{\circ}\right.$ ao $105^{\circ}$ dia de gestação $)$ é o momento em que o feto cresce lentamente, sendo o período ideal para a realização do diagnóstico de gestação. Em primeiro lugar, para confirmá-la. Em segundo, para identificar fêmeas com problemas reprodutivos e diagnosticar as causas (Gearhart et al., 1988). Em terceiro lugar, para que o proprietário possa planejar ajustes na dieta e divisão de lotes das fêmeas conforme o número de crias e, assim, se programar para receber os cordeiros que irão nascer.

O final da gestação (105 dias até o nascimento) é a fase que requer maiores cuidados com as ovelhas, devido ao maior desenvolvimento fetal, de seus envoltórios e da glândula mamária da fêmea (Hild et al., 2011; Coulon et al., 2013). Outra preocupação importante é a vacinação contra clostridioses entre a $4^{\circ}$ e a $6^{\circ}$ semana antes da data prevista do parto. Esta promoverá imunidade materna, que será 
passada ao filhote pelo colostro como primeira defesa até a vacinação dos cordeiros entre 6 e 8 semanas de vida (Fthenakis et al., 2012).

Neste período, a desnutrição pode comprometer o comportamento materno-filial pós-nascimento e a sobrevivência da cria (Dwyer et al., 2003). Os mesmos autores descobriram que níveis moderados de desnutrição comprometem os comportamentos maternais, como o grooming (movimento de lamber e mordiscar direcionados ao cordeiro). Também observaram uma redução na ligação materno-filial que, aliada ao baixo peso ao nascimento do cordeiro e desempenho lactacional da ovelha, reduzem as chances de sobrevivência do cordeiro. Uma explicação para as mudanças no comportamento maternal das ovelhas em desnutrição está relacionada ao aumento dos níveis de progesterona e à redução do estradiol entre o meio e o final da gestação (Dwyer et al., 2003).

Sob o ponto de vista nutricional, é no terço final da gestação e no início da lactação que a fêmea demanda maior aporte energético e proteico para produção e mantença (NRC, 2007). A suplementação com alimentos concentrados, pastagem ou forragens conservadas pode ser uma boa alternativa neste período, quando ofertados em qualidade e quantidade necessárias (Rocha et al., 2018).

Efetuar a adição gradual de concentrado na dieta das ovelhas contribuirá para a adaptação da microbiota ruminal. Como o requerimento energético aumenta neste período, aconselhase acompanhar o escore de condição corporal e verificar possíveis problemas nutricionais que estão afetando fêmeas. Sabe-se que a má nutrição nesta fase contribui para a ocorrência de desordens metabólicas, como a toxemia da gestação. Esta desordem é ocasionada pelo balanço energético negativo, devido ao menor espaço físico do rúmen pelo crescimento do feto e maior exigência nutricional (Rook, 2000), gliconeogênese hepática e elevação dos corpos cetônicos circulantes que deprimem o sistema nervoso central e os fetos (West, 1996; Van Saun, 2000). Vale ressaltar que as exigências nutricionais nos dois últimos meses de gestação aumentam de forma considerável, podendo chegar a $175 \%$ dos requerimentos de animais não gestantes, considerando o mesmo peso corporal (NRC, 2007).
Alterações fisiológicas como o aumento da concentração sanguínea de cortisol e temperatura retal foram observados por Henrique et al. (2017) ao trabalharem com injeção de lipopolissacarídeo (LPS - Escherichia coli $0,8 \mu \mathrm{g} \cdot \mathrm{kg}^{-1}$ de peso vivo) como simulador inflamatório e ativador do sistema imunológico (estresse sistêmico) em ovelhas no terço final de gestação, ocasionando maiores prejuízos no desenvolvimento destes cordeiros após o nascimento. Jensen et al. (2002), após submeterem ovelhas no terço final de gestação a estresse crônico por meio da infusão de hidrocortisona por 10 dias, constataram redução no crescimento fetal e alterações nas funções cardiovasculares, endócrinas e metabólicas dos fetos, com consequências negativas a longo prazo para as crias.

Alterações no sistema nervoso central, em decorrência de desafios no período fetal, podem acarretar em consequências negativas aos cordeiros, como desarranjos cognitivos, deficiência de memória e aprendizado (Coulon et al., 2015). Além disso, Hoffman et al. (2018) constataram que ovelhas em restrição nutricional no final da gestação apresentaram fetos com hipoglicemia, concentração sanguínea de IGF-1 alterada e comprometimento do desenvolvimento reprodutivo do neonato, com consequências negativas no desempenho reprodutivo das crias.

0 conforto térmico é outro ponto a ser considerado durante o período gestacional. Em países de clima tropical e subtropical, o estresse térmico promove mudanças metabólicas, fisiológicas e endócrinas que comprometem a produção e reprodução. Para manter a homeotermia, as ovelhas reduzem a ingestão de matéria seca, aumentam o consumo de água, elevam a frequência respiratória e a temperatura retal (Silva, 2000; Marai et al., 2007). A termorregulação nos ovinos ainda depende da espécie e da raça (Dangi et al., 2014; Dias e Silva et al., 2016). As raças nativas de países tropicais apresentam maior adaptabilidade ao calor, sendo mais tolerantes a este (Silva et al., 2017). Como mecanismos de termólise, as fêmeas gestantes se utilizam da polipneia térmica e aumento da frequência cardíaca (Yaqub et al., 2017), havendo, por consequência, diminuição do desempenho produtivo (Sejian et al., 2010) e do bem-estar (Maurya et al., 2016). 
Segundo Bell et al. (1989), as mudanças biológicas em decorrência do estresse por calor retardam o crescimento fetal e placentário, bem como reduzem a secreção de lactogênio e progesterona placentários. No feto, a transferência de calor ocorre por meio da circulação sanguínea materno-fetal, o que contribui para o incremento de calor para a fêmea gestante (Walker et al., 1995). Sendo assim, neste período o estresse térmico tem efeitos mais pronunciados tanto na ovelha quanto nos fetos, podendo acarretar o nascimento de cordeiros mais leves e menos desenvolvidos, o que eleva as chances de mortalidade (Brown et al., 1977; Hopkins et al., 1980). A termorregulação em cordeiros recém-nascidos pode ser influenciada pelo meio e também pela temperatura materna durante a gestação (McCrabb et al., 1993). Os mesmos autores observaram que cordeiros expostos ao estresse por calor durante a fase fetal, nascidos de ovelhas que mantiveram a temperatura retal menor em situações adversas, apresentaram maior peso ao nascer.

Dessa forma, o estresse térmico durante a fase gestacional compromete o desempenho materno e fetal com consequências após o nascimento dos cordeiros. Por isso, providenciar sombreamento e água de qualidade nas pastagens e áreas de descanso é de fundamental importância para esta categoria.

A consultoria técnica é de relevante contribuição neste período pelo levantamento de pontos críticos, planejamento de melhorias e execução de práticas que favoreçam os bons cuidados das ovelhas gestantes e o nascimento dos cordeiros.

\section{Conclusão}

A gestação das ovelhas é uma etapa crítica, pois diferentes mudanças biológicas transcorrem desde o início até o terço final, requerendo cuidados em cada fase para enfim alcançar o nascimento de cordeiros saudáveis. Prioriza-se o bom manejo nutricional, o acompanhamento da condição corporal, o conforto térmico, e evitam-se manejos que estressem os animais principalmente nas fases mais críticas da gestação, a inicial e a final. Muito progresso tem sido conquistado nas pesquisas recentes, por evidenciarem as consequências do estresse prénatal no desempenho e nas funções biológicas dos cordeiros, fornecendo, dessa forma, mais argumentos a favor do bem-estar adequado durante esta fase tão importante para o sucesso da ovinocultura.

\section{Referências}

Alexander G, Walser EES. Visual discrimination between ewes by lambs. Appl Anim Ethol. 1978;4(1):81-5.

Arnold GW, Morgan PD. Behaviour of the ewe and lamb at lambing and its relationship to lamb mortality. Appl Anim Ethol.1975;2(1):25-46.

Arnold GW, Wallace SR, Maller RA. Some factors envolved in natural weaning processes in sheep. Appl Anim Ethol. 1979;5(1):43-50.

Baldwin BA, Meese GB. The ability of sheep to distinguish between conspecifics by means of olfaction. Physiol Behav. 1977;18(5):803-8.

Banks EM. Some aspects of sexual behavior in domestic sheep, Ovis Aries. Behaviour. 1964;23(3/4):249-79.

Bell AW, McBride BW, Slepetis R, Early RJ, Currie WB. Chronic heat stress and prenatal development in sheep: I. Conceptus growth and maternal plasma hormones and metabolites. J Anim Sci. 1989;67(12):3289-99.

Berger J. Group size, foraging and antipredator ploys: An analysis of bighorn sheep decisions. Behav Ecol Sociobiol. 1978;4(1):91-9.

Braun K, Bock J, Wainstock T, Matas E, Gaisler-Salomon I, Fegert J, et al. Experience-induced transgenerational (re-)programming of neuronal structure and functions: Impact of stress prior and during pregnancy. Neurosci Biobeh Rev. 2017.

Broom DM. The effects of land transport on animal welfare. Rev Sci Tech. 2005;24(2):683-91.

Brown DE, Harrison PC, Hinds FC, Lewis JA, Wallace $\mathrm{MH}$. Heat stress effects on fetal development during late gestation in the ewe. J Anim Sci. 1977;44(3):442-6. 
Caroprese M. Sheep housing and welfare. Small Rumin Res. 2008;76(1-2):21-5.

Clutton-Brock TH, Albon SD, Guinness FE. Great expectations: dominance, breeding success and offspring sex ratios in red deer. Anim Behav. 1986;34(2):460-71.

Clutton-Brock JA. Natural history of domesticated mammals. Cambridge: Cambridge University Press;1999.

Coulon M, Nowak R, Andanson S, Petit B, Lévy F, Boissy A. Effects of prenatal stress and emotional reactivity of the mother on emotional and cognitive abilities in lambs. Dev Psychobiol. 2015;57(5):626-36.

Coulon M, Wellman CL, Marjara IS, Janczak AM, Zanella AJ. Early adverse experience alters dendritic spine density and gene expression in prefrontal cortex and hippocampus in lambs. Psychoneuroendocrinology. 2013;38(7):1112-21.

Dangi SS, Gupta M, Nagar V, et al. Impact of short-term heat stress on physiological responses and expression profile of HSPs in Barbari goats. Int J Biometeorol. 2014; 58: 2085-93.

Dias e Silva TP, Torreão JNC, Marques CAT, Araújo MJ, Bezerra LR, Dhanasekaran DK, et al. Effect of multiple stress factors (thermal, nutritional and pregnancy type) on adaptive capability of native ewes under semi-arid environment. J Therm Biol. 2016;59:39-46.

Doyle RE, Freire R, Cowling A, Knott SA, Lee C. Performance of sheep in a spatial maze is impeded by negative stimuli. Appl Anim Behav Sci. 2014;151:36-42.

Dumont B, Boissy A. Grazing behaviour of sheep in a situation of conflict between feeding and social motivations. Behav Processes. 2000;49(3):131-8.

Dwyer CM, Lawrence AB, Bishop SC, Lewis M. Ewelamb bonding behaviours at birth are affected by maternal undernutrition in pregnancy. $\mathrm{Br} \mathrm{J}$ Nutr. 2003;89(1):123-36.

Ewbank R. Nursing and suckling behaviour amongst Clun Forest ewes and lambs. Anim Behav. 1967;15(2): 251-8.
Færevik G, Andersen IL, Bøe KE. Preferences of sheep for different types of pen flooring. Appl Anim Behav Sci. 2005;90(3-4):265-76.

Fazio F, Arfuso F, Rizzo M, Giannetto C, Giudice E, Zanghì

$\mathrm{E}$, et al. Livestock handling and road transport influence some oxidative stress parameters in ewes. J Vet Behav. 2018;26:5-10.

Festa-Bianchet M. The social system of bighorn sheep: grouping patterns, kinship and female dominance rank. Anim Behav. 1991;42(1):71-82.

Fitzpatrick J, Scott M, Nolan A. Assessment of pain and welfare in sheep. Small Rumin Res. 2006;62(1-2):55-61.

Fitzpatrick RJ, Dobson H. The cervix of the sheep and goat during parturition. Anim Reprod Sci. 1979;2(1-3):209-24.

Fraser D. Animal Welfare and the Intensification of Animal Production. In: Thompson PB (E.). The Ethics of Intensification. The International Library of Environmental, Agricultural and Food Ethics. vol. 16. Dordrecht: Springer; 2008; p. 167-89.

Freitas ACB, Quirino CR, Bastos R. Bem-estar de ovinos: Revisão. Pubvet. 2017;11(1):18-29.

Fthenakis GC, Arsenos G, Brozos C, Fragkou IA, Giadinis ND, Giannenas I, et al. Health management of ewes during pregnancy. Anim Reprod Sci. 2012;130(3-4):198-212.

Gardner DS, Buttery PJ, Daniel Z, Symonds ME. Factors affecting birth weight in sheep: maternal environment. Reproduction. 2007;133(1):297-307.

Gearhart MA, Wingfield WE, Knight AP, Smith JA, Dargatz DA, Boon JA, et al. Real-time ultrasonography for determining pregnancy status and viable fetal numbers in ewes. Theriogenology. 1988;30(2):323-37.

Goddard P, Waterhouse T, Dwyer C, Stott A. The perception of the welfare of sheep in extensive systems. Small Rum Res. 2006;62(3):215-25.

Grandin T. Livestock handling and transport. 3 ed. Wallingford: CABI International; 2007. 
Hafez B, Hafez ESE. Reproduction in farm animals. 7 ed. Filadélfia: Lippincott Williams \& Wilkings; 2000.

Henrique FL, Titto EAL, Zanella AJ, Hooper HB, PulidoRodriguez LF, Longo ALS, et al. Simulated disease process during late pregnancy compromises developmental outcomes of lambs independently of the weaning method applied. Small Rum Res. 2017;155:39-44.

Hild S, Andersen IL, Zanella AJ. The relationship between thermal nociceptive threshold in lambs and ewe-lamb interactions. Small Rum Res. 2010;90(1-3):142-5.

Hild S, Clark CCA, Dwyer CM, Murrell JC, Mendl M, Zanella AJ. Ewes are more attentive to their offspring experiencing pain but not stress. Appl Anim Behav Sci. 2011;132(3-4):114-20.

Hoffman F, Boretto E, Vitale S, Gonzalez V, Vidal G, Pardo MF, et al. Maternal nutritional restriction during late gestation impairs development of the reproductive organs in both male and female lambs. Theriogenology. 2018;108:331-8.

Hopewell L, Rossiter R, Blower E, Leaver L, Goto K. Grazing and vigilance by Soay sheep on Lundy island: Influence of group size, terrain and the distribution of vegetation. Behav Processes. 2005;70(2):186-93.

Hopkins PS, Nolan CJ, Pepper PM. The effects of heat stress on the development of the foetal lamb. Aust J Agric Res. 1980;31(4):763-71.

Hulet CV, Alexander G, Hafez ESE. The behaviour of sheep. In: Hafez ESE (E.). The Behaviour of Domestic Animals. Londres: Bailliere Tindall; 1975.

IBGE. Participação das regiões no rebanho ovino, 2007 e 2016. [acesso 27 fev 2018]. Disponível em: https:// tinyurl.com/y7grxqsb.

Jensen EC, Gallaher BW, Breier BH, Harding JE. The effect of a chronic maternal cortisol infusion on the lategestation fetal sheep. J Endocrinol. 2002;174(1):27-36.

Keeling LJ, Gonyou HW. Social behaviour in farm animals. Nova York: CABI Publishing; 2001. 406 p.
Kendrick KM, Atkins KA, Hinton M, Heavens P, Keverne BE. Are faces special for sheep? Evidence from facial and object discrimination learning tests showing effects of inversion and social familiarity. Behav Processes. 1996;38(1):19-35.

Kumar D, Sejian V, Gaughan JB, Naqvi SMK. Biological functions as affected by summer season-related multiple environmental stressors (heat, nutritional and walking stress) in Malpura rams under semiarid tropical environment. Biol Rhythm Res. 2017;48(4):593-606.

Levine S. Primary social relationships influence the development of the hypothalamic-pituitary-adrenal axis in the rat. Physiol Behav. 2001;73(3):255-60.

Levine S. Regulation of the hypothalamic-pituitaryadrenal axis in the neonatal rat: the role of maternal behavior. Neurotox Res. 2002;4(5-6):557-64.

Levine S, Alpert M, Lewis GW. Differential maturation of an adrenal response to cold stress in rats manipulated in infancy. J Comp Physiol Psychol. 1958;51(6):774-7.

Levy F, Poindron P. The importance of amniotic fluids for the establishment of maternal behaviour in experienced and inexperienced ewes. Anim Behav. 1987;35(4):1188-92.

Lynch JJ, Hinch GN, Adams DB. The behaviour of sheep: biological principles and implications for production. Wallingford: CAB International; 1992.

Madruga MS, Sousa WH, Rosales MD, Cunha MGG, Ramos JLF. Qualidade da carne de cordeiros Santa Inês terminados com diferentes dietas. R Bras Zootec. 2005;34(1):309-15.

Marai IFM, El-Darawany AA, Fadiel A, Abdel-Hafez MAM. Physiological traits as affected by heat stress in sheep-A review. Small Rumin Res. 2007;71(1-3):1-12.

Matthews SG. Early programming of the hypothalamopituitary-adrenal axis. Trends Endocrinol Metab. 2002;13(9):373-80. 
Maurya VP, Sejian V, Kumar D, Naqvi SMK. Impact of heat stress, nutritional restriction and combined stresses (heat and nutritional) on growth and reproductive performance of Malpura rams under semi-arid tropical environment. J Anim Physiol Anim Nutr (Berl). 2016;100(5):938-46.

McCrabb GJ, McDonald BJ, Hennoste LM. Heat stress during mid-pregnancy in sheep and the consequences for placental and fetal growth. J Agric Sci. 1993;120(2):265-71.

Menzies P. The Behaviour of Sheep - Biological Principles and Implications for Production. Can Vet J. 1994;35(1):24.

Molle G, Branca A, Ligios S, Sitzia M, Casu S, Landau $S$, et al. Effect of grazing background and flushing supplementation on reproductive performance in Sarda ewes. Small Rumin Res. 1995;17(3):245-54.

More S, Bicout D, Botner A, Butterworth A, Calistri P, Depner K, et al. Animal welfare aspects in respect of the slaughter or killing of pregnant livestock animals (cattle, pigs, sheep, goats, horses). EFSA J. 2017; 5(5):4782.

Mori RM, Ribeiro ELA, Mizubuti IY, Rocha MA, Silva LDF. Desempenho reprodutivo de ovelhas submetidas a diferentes formas de suplementação alimentar antes e durante a estação de monta. R Bras Zootec. 2006;35(3):1122-8.

Munoz C, Campbell A, Hemsworth P, Doyle R. AnimalBased Measures to Assess the Welfare of Extensively Managed Ewes. Animals (Basel). 2018;8(1):2.

Naqvi SMK, Sejian V, Karim SA. Effect of feed flushing during summer season on growth, reproductive performance and blood metabolites in Malpura ewes under semiarid tropical environment. Trop Anim Health Prod. 2012;45(1):143-8.

Nowak R, Boivin X. Filial attachment in sheep: Similarities and differences between ewe-lamb and human-lamb relationships. Appl Anim Behav Sci. 2015;164:12-28.
Nowak R, Porter RH, Lévy F, Orgeur P, Schaal B. Role of mother-young interactions in the survival of offspring in domestic mammals. Rev Reprod. 2000;5(3):153-63.

NRC. Nutrient Requirements of Small Ruminants: Sheep, Goats, Cervids, and New World Camelids. Washington, D.C.: The National Academies Press; 2007. 384 p.

O'Connor CE, Lawrence AB. Relationship between lamb vigour and ewe behaviour at parturition. Anim Prod. 1992;54(3):361-6.

Pereira ES, Campos ACN, Castelo-Branco KF, Bezerra LR, Gadelha CRF, Silva LP, et al. Impact of feed restriction, sexual class and age on the growth, blood metabolites and endocrine responses of hair lambs in a tropical climate. Small Rumin Res. 2018;158:9-14.

Pérez-Barbería FJ, Walker DM. Dynamics of social behaviour at parturition in a gregarious ungulate. Behav Processes. 2018;150:75-84.

Pérez-Torrero E, Rubio-Navarro L. Maternal behavior their adjustments and implicated factors. J Behav Brain Sci. 2015;5(2):40-55.

Pinheiro AA, Brito IF. Bem-estar e Produção Animal. Sobral: Embrapa Caprinos e Ovinos; 2009. 25 p.

Pires CC, Silva LF, Schlick FE, Guerra DP, Biscaino G, CarneiroRM. Growing and finishing of feedlot lambs. Cienc Rural. 2000;30(5):875-80.

Rech CLS, Rech JL, Fischer V, Osório MTM, Manzoni N, Moreira HLM, et al. Temperamento e comportamento materno-filial de ovinos das raças Corriedalee Ideal e sua relação com a sobrevivência dos cordeiros. Cienc Rural. 2008;38(5):1388-93.

Rocha AM, Dias e Silva TP, Sejian V, Torreão JNC, Marques CAT, Bezerra LR, et al. Maternal and neonatal behavior as affected by maternal nutrition during prepartum and postpartum period in indigenous sheep. J Vet Behav. 2018;23:40-6.

Rook JS. Pregnancy toxemia of Ewes, Does and Beef Cows. Vet Clin North Am Food Anim Pract. 2000;16(2):293-317. 
Rosa HJD, Bryant MJ. Seasonality of reproduction in sheep. Small Rumin Res. 2003;48(3):155-71.

Rutherford KMD. Assessing Pain in Animals. Anim Welf. 2002;11(1):31-53.

Sejian V, Maurya VP, Naqvi SMK. Adaptive capability as indicated by endocrine and biochemical responses of Malpura ewes subjected to combined stresses (thermal and nutritional) in a semi-arid tropical environment. Int J Biometeorol. 2010;54(6):653-61.

Sevi A, Caroprese M. Impact of heat stress on milk production, immunity and udder health in sheep: A critical review. Small Rumin Res. 2012;107(1):1-7.

Silva RG. Introdução à bioclimatologia animal. São Paulo: Nobel; 2000. 286 p.

Silva WE, Leite JHGM, Sousa JER, Costa WP, Silva WST, Guilhermino MM, et al. Daily rhythmicity of the thermoregulatory responses of locally adapted Brazilian sheep in a semiarid environment. Int J Biometeorol. 2017;61(7):1221-31.

Stefanakis A, Volanis M, Zoiopoulos P, Hadjigeorgiou I. Assessing the potential benefits of technical intervention in evolving the semi-intensive dairy-sheep farms in Crete. Small Rumin Res. 2007;72(1):66-72.

Thornton PD, Waterman-Pearson AE. Behavioural Responses to Castration in Lambs. Anim Welf. 2002; 11(2):203-12.
Tur I, Dínç DA, Semacan A. Protein based flushing related blood urea nitrogen effects on ovarian response, embryo recovery and embryo quality in superovulated ewes. Theriogenology. 2017;98:62-7.

Van Saun RJ. Pregnancy toxemia in a flock of sheep. J Am Vet Med Assoc. 2000;217(10):1536-9.

Walker DW, Hale JR, Fawcett A, Pratt NM. Cardiovascular responses to heat stress in late gestation fetal sheep. Exp Physiol. 1995;80(5):755-66.

Wang YQ, Zhong RZ, Fang Y, Zhou DW. Influence of tail docking on carcass characteristics, meat quality and fatty acid composition of fat-tail lambs. Small Rumin Res. 2018;162:17-21.

West HJ. Maternal undernutrition during late pregnancy in sheep. Its relationship to maternal condition, gestation length, hepatic physiology and glucose metabolism. Br J Nutr. 1996;75(4):593-605.

Whitteridge D. The development of the visual system in the sheep. Arch Ital Biol. 1978; 116 (3-4): 406-408.

Yaqub LS, Ayo JO, Kawu MU, Rekwot PI. Diurnal thermoregulatory responses in pregnant Yankasa ewes to the dry season in a tropical Savannah. Trop Anim Health Prod. 2017; 49: 1243-1252.

Zohary D, Tchernov E, Horwitz L. The role of unconscious selection in the domestication of sheep and goats. J Zool. 1998; 245 (2): 129-135. 